\title{
Twinkle Megane: Near-Eye LED Indicators on Glasses in Tele-Guidance for Elderly
}

\author{
Yukitoshi Kashimoto ${ }^{1}$, Aryan Firouzian ${ }^{2}$, Zeeshan Asghar $^{2}$, Goshiro Yamamoto ${ }^{1}$, Petri Pulli ${ }^{2}$ \\ ${ }^{1}$ Nara Institute of Science and Technology, 8916-5 Takayama-cho, Ikoma city, Nara, Japan \\ ${ }^{2}$ University of Oulu, Pentti Kaiteran katu 1, 90570, Oulu, Finland \\ Email: ${ }^{1}$ (kashimoto.yukitoshi.km3, goshiro)@is.naist.jp, ${ }^{2}$ (aryan.firouzian, zeeshan.asghar, petri.pulli)@oulu.fi
}

\begin{abstract}
The development of wearable technologies has generated significant interest in the last decades. Considering global social issues such as aged society besides the technologies, senior citizens suffering from memory loss would be one of potential user groups. Since the use of eyeglasses among the elderly is quite common, we have constructed a navigation interface on eyeglasses by implanting a set of light emitting diode indicators on the frame of the glasses. We aim to realize eyeglasses-based navigation system that provides the elderly with the visual cues and could be interpreted intuitively as navigational commands. Furthermore, we believe that our system would improve the independent mobility of the elderly. This paper explains our first prototype glasses with near-eye LED indicators, incremental experimentation, and the preliminary results that are used to optimize the visual cues for real navigational tasks. The system was evaluated in simulated navigational tasks by the elderly participants suffering from dementia. We assessed our system by he experimentation and living lab methodology with data-driven approach.
\end{abstract}

\section{INTRODUCTION}

The emergence of wearable technologies in the last decade aims to ease human daily life by facilitating the humancomputer interaction. The proposition that the elderly should be considered as a potential user group of these emerging technologies is a provocative one, and one that we believe in. To subscribe to the belief that the older adults can use these technologies as efficient as youth is irrational. It has derived many researchers to design sympathetic customized devices to assist senior citizens in their daily tasks. Considering the population of elderly suffering from memory loss and cognitive decline, this paper aims to design a wearable navigational tool to assist them in their simple daily navigational tasks.

Bharucha et al. (2009) estimated 28 million people suffered from dementia in 2009, and it costs 156 billion dollars annually for caretaking them directly [1]. Furthermore, Ferri et al. (2006) estimated that more than 20 million people suffered from different type of dementia in the year 2005, and demented population increases by 4.6 million people every year [2]. The analysis of the older adults demographic suffering from memory loss has drawn attention to the use of technologies, in order to involve less human and financial resources in the caretaking process.

Zakzanis et al. (2009) conducted a study to investigate effects of age, gender and dementia on navigation and dexterity ability of people. The result shows dementia such as Alzheimers disease deteriorate the potential abilities in the spatial navigation tasks and using functional tasks such as working with the smart phones [3]. Sorri et al. (2011) conducted a set of navigation experiments with people suffering from memory loss. The subjects received visual, audio and tactile signals in three different modules to walk in different directions. The visual cues and the audio messages made the most efficient guidance to the subjects while the tactile signs were not as efficient as the visual cues and the audio messages [4].

Nowadays, new technologies are inevitably involved in daily life of the senior citizens. Wilpon et al. (1996) believed positive attitude of the senior citizens toward a technology would facilitate the use of them in their daily life [5]. Since the use of eyeglasses is very common among senior citizens, wearable smart glasses with the ability to assist the elderly in their daily navigational task can provide convenient guidance to them. The older adults usually have the positive attitude toward technology, and they want it to ease their daily life tasks. However, the user interface interaction is the main problem for them [6]. A wearable device with a headmounted display would leave hands free, and provide users with information on the near eye display [7]. The wearable technology could be improved by implementing the platform for sensing, monitoring and processing data while increasing comfortability and convenience [8].

In the recent years, some of the industrial manufacturers such as Google and Epson have provided the users with niche wearable products. Google manufactured the high-tech Google Glass to be replaced as a wearable smartphone. It has demonstrated high potential for practical use in educational and medical settings. Sedgwick (2014) conducted an industrial study on the use of Google Glass as a cost-effective collision warning and navigation tool in the driving tasks. The study unveiled controversial issues that threaten the safety while using the device in the navigational tasks [9]. Danton (2014) stated that users need to focus on the small display on the up-right corner of the eyeglasses' lens to get the information. It needs user's foreground of attention, and it stays out of the direct field of the vision. It may cause distraction in situation, which user needs the full attention in the physical environment [10]. Furthermore, Kunze et al. (2014) conducted an explorative study on the use of Google Glass in the daily life routine of the older adults. They stated that the current size of the display is not eligible enough for the senior citizens to 


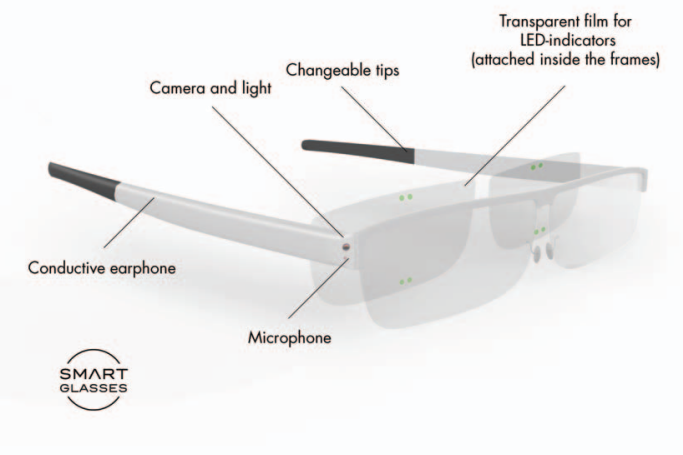

Fig. 1. The conceptual design of the device includes essential requirements.

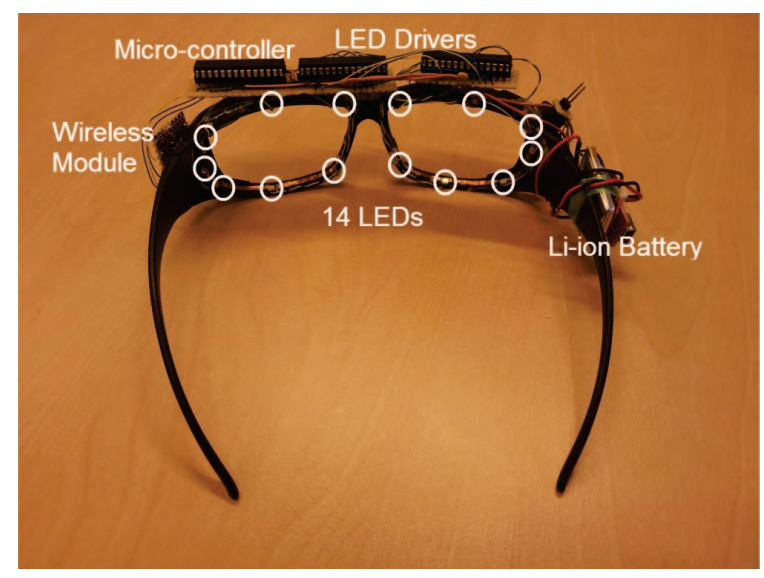

Fig. 2. The constructed prototype. White circles depict the position of LED indicators implanted on the frame of the eyeglasses.

read the information from it clearly [11]. Epson Moverio Smart Glasses provide users with a head up transparent display, which is more efficient in the navigational tasks since the users could still keep the foreground of attention on the physical environment [12]. However, use of head-up display for the senior citizens suffering from dementia can be challenging. The augmented reality images may completely overlay the imagery display on the top of the field of view and mask the elderly user's vision.

In this study, an innovative concept is introduced to design a noble artifact for assisting senior citizens with simple daily navigational tasks. We have developed the first prototype of Indicator-based Smart glasses to provide users with the visual cues, which do not interfere with their vision on the physical environment. The users can focus on their task while being guided by the visual cues. The usability experiments demonstrate that the severity of dementia strongly affects performance of the senior citizens in the navigational tasks and the use of technologies.

\section{Concept And Prototype of SMart Glasses With NeAR-Eye Visual Cue IndicAtors}

The ideal conceptual design of the Indicator-based Glasses with integral components are shown in the Figure 1. The head mounted display includes peripheral components of the mounted camera, global positioning system (GPS) tracker, accelerometer, gyroscope, step detector sensors and Bluetooth headset. A rechargeable battery provides the device with the electrical power. The Bluetooth headset facilitates the communication mechanism between the remote caretaker and the user. The implemented sensors collect detailed real-time information from user's environment for delivering to the remote caretaker. Integrated camera captures the picture that shows user field of view. Other essential raw data such as GPS coordinate, gyroscope and the step detector data facilitate and improve user localization for the remote caretaker.

Main users of the system are elderly people suffering from memory loss, and they need continuous assistance in simple navigational tasks. The required user interface should convey simple navigational commands to the users. Light Emitting Diode (LED) indicators are used in the mentioned HMD system for continuous navigational assistance. The LEDs are implanted in the frame of the glasses, and they can blink in different combination to provide navigational commands. The position of indicators on the frame are shown in the Figure 2. To provide various combinations of blinking indicator, seven indicators implanted on the frame of each lens. These indicators can blink in three different colors of red, green and yellow to generate navigational commands based on traffic light metaphor. Combinations of indicators with a different blinking pattern are designed to convey meaningful navigational cues. Usability experiments evaluate the efficiency of these visual cues and rate the different combination and pattern. The combination means several indicator blink simultaneously, and pattern means a set of setting in blinking frequency, light intensity and blinking time [13].

Figure 3 demonstrates the system diagram and the integral components of the system. A Lithium-ion battery generates stable $3.3 \mathrm{~V}$ and supplies electrical power for all the components. Android application is developed to set command pattern and trigger individual or combination indicators to blink. The application connects to the Bluetooth serial port profile (SPP) module on the device. The Android application sends the bit array data to the micro-controller. The SPP module delivers the data, which contains 32 bits binary data, to the micro-controller. Figure 4 depicts how the bit array should be decoded by the micro-controller. Twenty-eight bits represent the binary values to switch on or off the fourteen LEDs. Four bits represent the brightness of LEDs in the scale of 0 to 15 . First 28 bits are divided into fourteen pairs of bits. In the other word, each two bits are assigned to control each LED indicator. While the first bit is set, the LED indicator blinks in red and while the second bit is set and the first bit is unset, the LED indicator blinks in green. If both bits are set, the LED indicator blinks in yellow. The micro-controller 


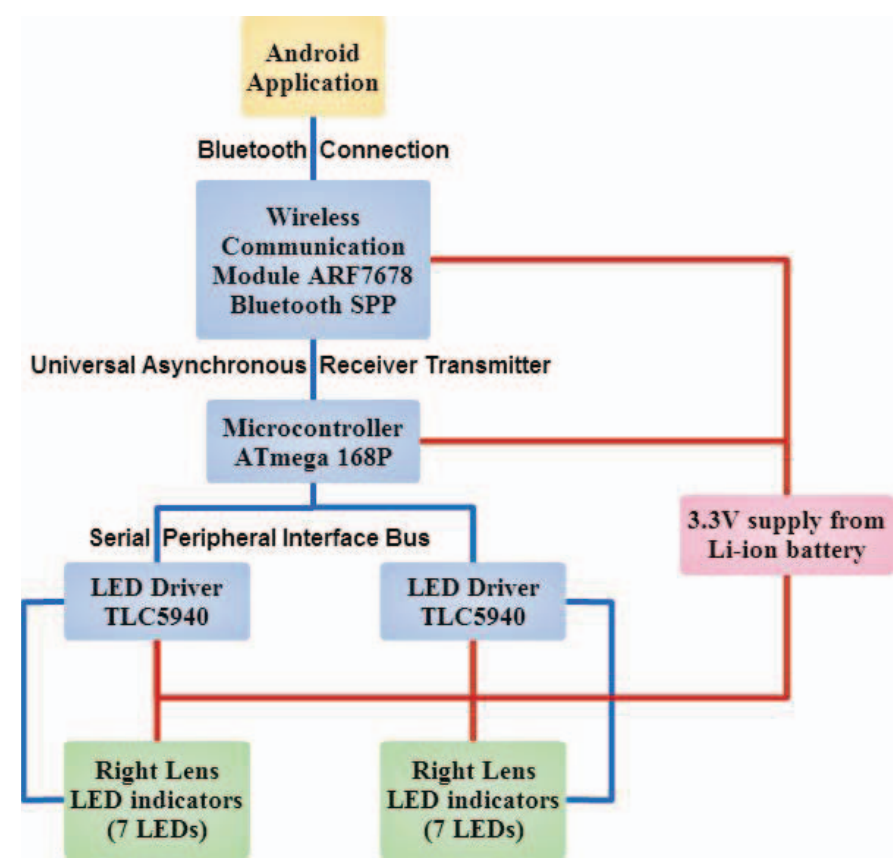

Fig. 3. The electronic components; circuit diagram illustrates connectivity of the components.

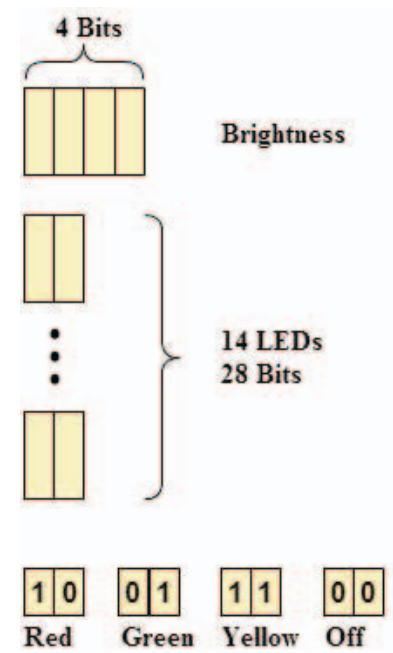

Fig. 4. The bit array data from the Android application

decodes the bit array data to controls 14 LEDs through two LED drivers [13].

To conduct usability experiments on the constructed prototype, two Android application are developed. Two applications communicate with the device as described earlier. The difference is the user interface of the application to set the blinking pattern for individual indicators and indicators combination as navigational cues. In the first Android application, different indicators with particular colours, brightness, and blinking time duration are set and sent to the device via Bluetooth connection. Figure 5 shows the graphical user interface of the application. In the second Android application, navigational buttons are included in the graphical user interface. Figure 6

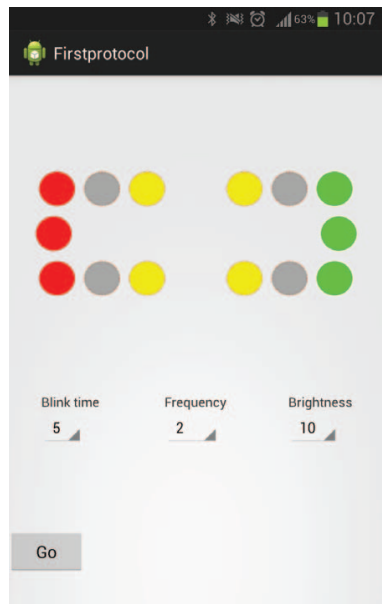

Fig. 5. The Android application sets indicator colors, frequency, brightness and blinking time and sends the visual cue to the device.

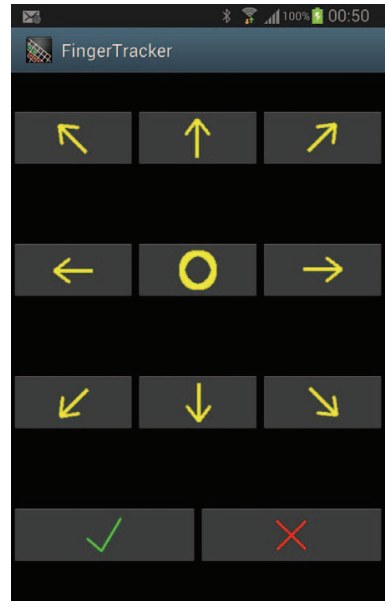

Fig. 6. The Android application sends visual commands to the device. Visual cue properties could be set in the setting menu. demonstrates the user interface of Android application to guide users. Specific visual cues could be assigned to each navigational button in the setting menu [13].

Based on the Wizard of $\mathrm{Oz}$ method, the application simulated the navigational commands from the remote caretaker unit or an automated assistant way-finding service. It provides the research study with the utility to conduct the usability experiments to evaluate user interface of the device by separating the HMD system from the tele-presence ecosystem. Initially, the most satisfactory blinking pattern are recognized in the experiment. It means the most convenient blinking frequency, brightness and blinking time. Then, in the next phase of the experiment, the most distinguishable combinations of indicators are recognized to convey simple turn-by-turn navigational commands. These visual cues are evaluated in the walking experiment to guide senior citizens reaching an end point in the indoor environment [13].

\section{USABILITY EXPERIMENTS}

The idea that experimentations evaluate usability of a design science prototype for real life situation is a grounded one, and one that we believe in. We used design science research method with qualitative and quantitative approach. Hevner et al. (2010) provided the guideline for the development of a design science artifact through iterative usability tests. The guideline suggests early pilot testing and iterative usability experiments with real users, to evaluate and improve the system [14]. For this purpose, we designed four-step experiments with different user groups. The Wizard of $\mathrm{Oz}$ method with unstructured interviews, living lab approach, and video recording are used to interpret the data [15].

Figure 7 shows the sequence of the experiments, and it specifies the measured factors and variables in the experiments. The first experiment is localizing indicators test, and it aims to evaluate the visibility of individual LED indicators on the frame. The indicators blink individually with specific 


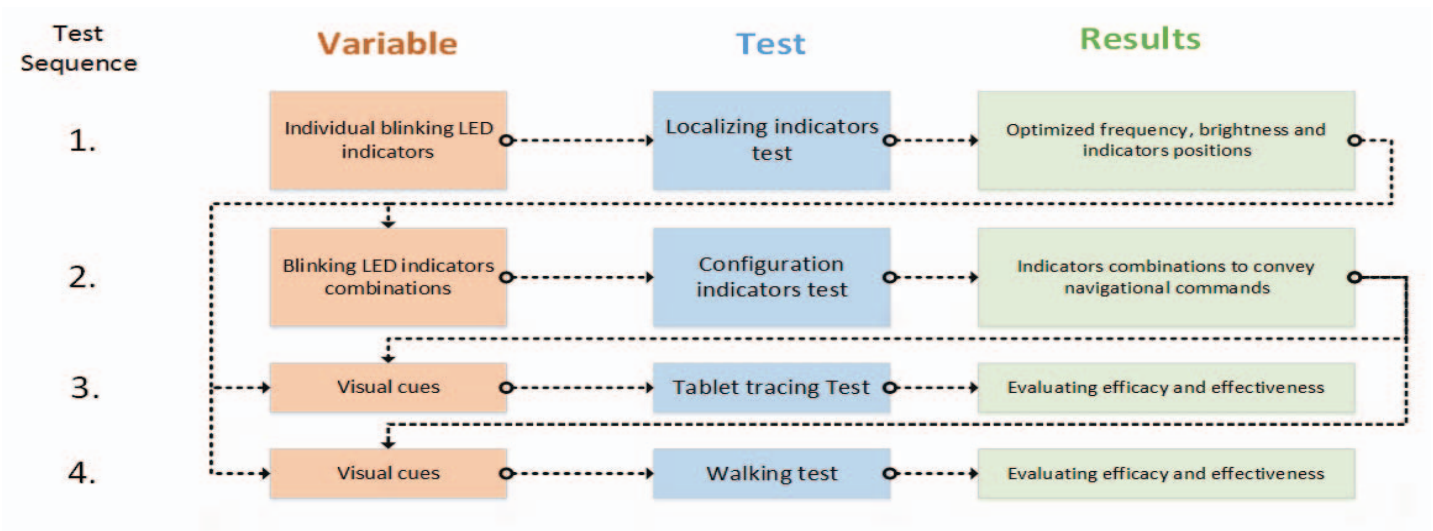

Fig. 7. The sequence of the four-step experiments. It shows how the results of the first two experiments would form the most distinguishable visual cues for the last two experiments.

alternatives of frequencies and brightness. The result demonstrated accuracy in recognizing individual blinking indicators. Bayesian analysis indicates what indicators on the frame might be missed when they are blinking, and what indicators might be mistakenly chosen as blinking [16]. In addition, the results clarify the satisfactory blinking pattern for blinking indicators including optimized brightness and frequency.

The second experiment is indicators configurations test. Different sets of LED indicators' combinations blink simultaneously, and users are supposed to choose interpreted navigational command such as left, right and so on. The results of the first experiment are applied to sets of combinations. It includes optimized frequency, optimized brightness, and most distinguishable indicators on the frame. The result of the second experiments demonstrates the most meaningful indicators configurations to convey navigational commands.

The third experiment is tablet-tracing test; users are supposed to move a stylus on a handheld device based on the navigational commands of the prototype. The results of the first experiment are applied to blinking indicators and the results of the second experiment, which are the most meaningful combination of the indicators as navigational commands, are used as visual cues. The main aim of the tablet-tracing test is to evaluate users' reactions to the visual cues. This experiment assures validity and efficiency of interpreted visual cues.

The last experiment simulates simple navigational tasks for the subjects in an indoor open environment. The users are supposed to walk on predefined map while the visual cues guide them. The map contains specific number of stops and turning left and right with different rotation degrees. The optimized frequency and brightness are applied to the blinking indicators, and the navigational commands are defined based on the results of the second experiment.

Three different user groups participated in the experiments. At first, four pilot tests with student users were conducted to confirm test protocols and validate the preliminary results. Then the system went through the usability experiments with the elderly suffering from severe dementia. Finally, the us- ability experiments with the senior citizens suffering from mild and moderate dementia were conducted. The ambient brightness is measured via illumination measurement device as 200-3001x in all the test environments. Figure 8 demonstrated the order of the conducted experiments with all the user groups.

\begin{tabular}{|l||c|c|c|}
\hline Variable & Subject PA & Subject PB & Subject PC \\
\hline $\begin{array}{l}\text { Number } \\
\text { commands }\end{array}$ & 28 & $28+9$ & 28 \\
\hline $\begin{array}{l}\text { Mistakes by sub- } \\
\text { jects }\end{array}$ & 0 & 9 & 0 \\
\hline $\begin{array}{l}\text { Correction via de- } \\
\text { vice }\end{array}$ & 0 & 9 & 0 \\
\hline $\begin{array}{l}\text { Correction via hu- } \\
\text { man assistant }\end{array}$ & 0 & 0 & 0 \\
\hline $\begin{array}{l}\text { Accomplishment } \\
\text { time }\end{array}$ & $76 \mathrm{~s}$ & $124 \mathrm{~s}$ & $83 \mathrm{~s}$ \\
\hline Average speed & $0.36 \mathrm{~m} / \mathrm{s}$ & $0.22 \mathrm{~m} / \mathrm{s}$ & $0.33 \mathrm{~m} / \mathrm{s}$ \\
\hline Path length & $27 \mathrm{~m}$ & $27 \mathrm{~m}$ & $27 \mathrm{~m}$ \\
\hline
\end{tabular}

THE RELEVANT FACTORS ARE MEASURED FOR THREE STUDENT SUBJECTS FOR WALKING IN AN OPEN ENVIRONMENT WHILE GUIDED BY VISUAL CUES.

Seventeen student subjects participated in the pilot tests with the average age of 26.05 and the range of 20 to 33, without problems in the vision ability. The results of the Bayesian analysis for the localizing indicators test unveiled lowest sensitivity and specificity for nasal indicators (indicators close to the nose). In other word, the nasal LED indicators can be missed while blinking, and they can be mistakenly considered as blinking while they are not. Therefore, we used less number of nasal LEDs in the formation of the visual cues. In the indicators configuration test, 48 forms of navigational cues (combination of blinking indicators) were shown to the subjects, and ten of them were chosen as the navigational commands based on the users' preferences. In the last two experiments, the student users accomplished their task by following the visual cues to move the stylus on the tablet device and walk the predefined route. Task accomplishment means moving from the starting point, following the predefined route 


\begin{tabular}{|l|c|c|c|c|}
\hline Variable & Subject A & Subject B & Subject C & Subject D \\
\hline Age & 81 & 80 & 83 & 74 \\
\hline Gender & Male & Male & Female & Male \\
\hline Using eyeglasses & No & Yes & Yes & Yes \\
\hline $\begin{array}{l}\text { Number of com- } \\
\text { mands }\end{array}$ & $28+6$ & $28+0$ & $28+3$ & $28+4$ \\
\hline $\begin{array}{l}\text { Mistakes by sub- } \\
\text { jects }\end{array}$ & 8 & 7 & 7 & 5 \\
\hline $\begin{array}{l}\text { Correction via } \\
\text { device }\end{array}$ & 6 & 0 & 3 & 4 \\
\hline $\begin{array}{l}\text { Correction via } \\
\text { human assistant }\end{array}$ & 2 & 7 & 4 & 1 \\
\hline $\begin{array}{l}\text { Accomplishment } \\
\text { time }\end{array}$ & $220 \mathrm{~s}$ & $390 \mathrm{~s}$ & $286 \mathrm{~s}$ & $136 \mathrm{~s}$ \\
\hline Average speed & $0.068 \mathrm{~m} / \mathrm{s}$ & $0.038 \mathrm{~m} / \mathrm{s}$ & $0.052 \mathrm{~m} / \mathrm{s}$ & $0.110 \mathrm{~m} / \mathrm{s}$ \\
\hline Path length & $15 \mathrm{~m}$ & $15 \mathrm{~m}$ & $15 \mathrm{~m}$ & $15 \mathrm{~m}$ \\
\hline
\end{tabular}

THE RELEVANT DESCRIPTIVE STATISTICS AND FACTORS ARE MEASURED FOR FOUR ELDERLY SUBJECTS SUFFERING FROM MILD AND MODERATE DEMENTIA. THE EXPERIMENT INCLUDES WALKING IN AN OPEN ENVIRONMENT WHILE GUIDED BY VISUAL CUES.

and reaching the end point. The results validate that the visual cues could be used as navigational commands. Table I shows the results of the last pilot experiment.

Fourteen elderly subjects participated in the usability experiments with the average age of 75.36 and the range of 61 to 91 . Nine subjects used eyeglasses for most of the working hours. The severity level of dementia is diagnosed via mini-mental state examination (MMSE) or the information of rehabilitation center. MMSE score less than 9/30 indicates severe dementia, and the score more than 9/30 represents mild and moderate dementia [17]. Most of the senior citizens with dementia suffered from the difficult experiences such as losing track of the topic and distractibility [18]. The severity of dementia deteriorates the abovementioned conditions [19]. Abovementioned difficulties prevented the elderly subjects to accomplish their tasks in experiments with subjects suffering from severe dementia. In addition, staying in a stationary position for a long time, focusing and using the handheld device and the stylus to follow the visual cues distract them in the tablet tracing experiments. In the localizing indicator experiment with elderly suffering from mild and moderate dementia, the subjects constantly responded to the visual cues by giving directions. Pointing to the exact position of indicators was difficult for the subjects. Therefore, the experiment failed to provide satisfactory data for analysis.

All the subjects suffering from mild and moderate dementia accomplished their tasks by following the visual cues and walking in the predefined route. Table II shows the most important collected data in the experiment. The subjects reacted more accurately to the visual cues at the end part of the walking task. It shows high adaptability to the system in performing the tasks. Figure 9 depicts the map of the navigation area in an open indoor environment. The subjects had to follow the system commands without predicting the route. Furthermore, in real life situations people need navigational guidance in the junctions and more clear situations. The indicators were blinking with frequency of $1 \mathrm{~Hz}, 1,5 \mathrm{~Hz}$

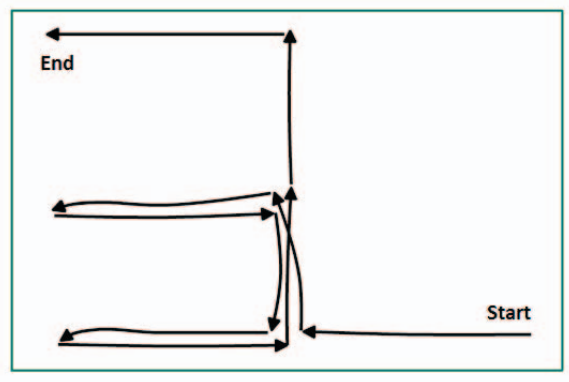

Fig. 9. The predefined map contains number of rotations to the left and right in an open area.

and $2 \mathrm{~Hz}$ in the pilot and usability experiments. The subjects achieved the best results while indicators were blinking with frequency of $1 \mathrm{~Hz}$. Nevertheless, the unstructured interviews uncovered that the elderly adults prefer the frequency less than $1 \mathrm{~Hz}$. In the indicators configuration experiment, 32 forms of navigational cues are shown to the elderly suffering from mild and moderate dementia, and they chose navigational commands for each of them. The test results mostly approved the results of the pilot experiment for the meaningful indicators combinations to convey the navigational commands.

\section{CONCLUSION}

The usability experiments demonstrate that the severity of dementia strongly affects performance of the senior citizens in the navigational tasks and the use of technologies. The subject's experience in using the navigation tools is another salient factor; for example, some of the subjects considered that turning left is always a 90-degree turn while other turned left until the command continued. Furthermore, customizing the navigational commands based on the users preferences is a promising solution. The unstructured interviews revealed that the senior citizens are mostly interested in using the device. However, they complained about the weight of the device. Furthermore, we consider constructing new lightweight prototype with mounted cameras and localizing sensors. In addition, to customize and improve the system, the prototype needs to be evaluated in the iterative usability experiments.

\section{REFERENCES}

[1] A. J. Bharucha, V. Anand, J. Forlizzi, M. A. Dew, C. F. Reynolds, S. Stevens, and H. Wactlar, "Intelligent assistive technology applications to dementia care: current capabilities, limitations, and future challenges," The American journal of geriatric psychiatry, vol. 17, no. 2, pp. 88-104, 2009.

[2] C. P. Ferri, M. Prince, C. Brayne, H. Brodaty, L. Fratiglioni, M. Ganguli, K. Hall, K. Hasegawa, H. Hendrie, and Y. Huang, "Global prevalence of dementia: a delphi consensus study," The Lancet, vol. 366, no. 9503, pp. 2112-2117, 2006.

[3] K. K. Zakzanis, G. Quintin, S. J. Graham, and R. Mraz, "Age and dementia related differences in spatial navigation within an immersive virtual environment," Medical science monitor : international medical journal of experimental and clinical research, vol. 15, no. 4, pp. CR14050, Apr 2009, jID: 9609063; ppublish.

[4] L. Sorri, E. Leinonen, and M. Ervasti, "Wayfinding aid for the elderly with memory disturbances." in ECIS, 2011. 


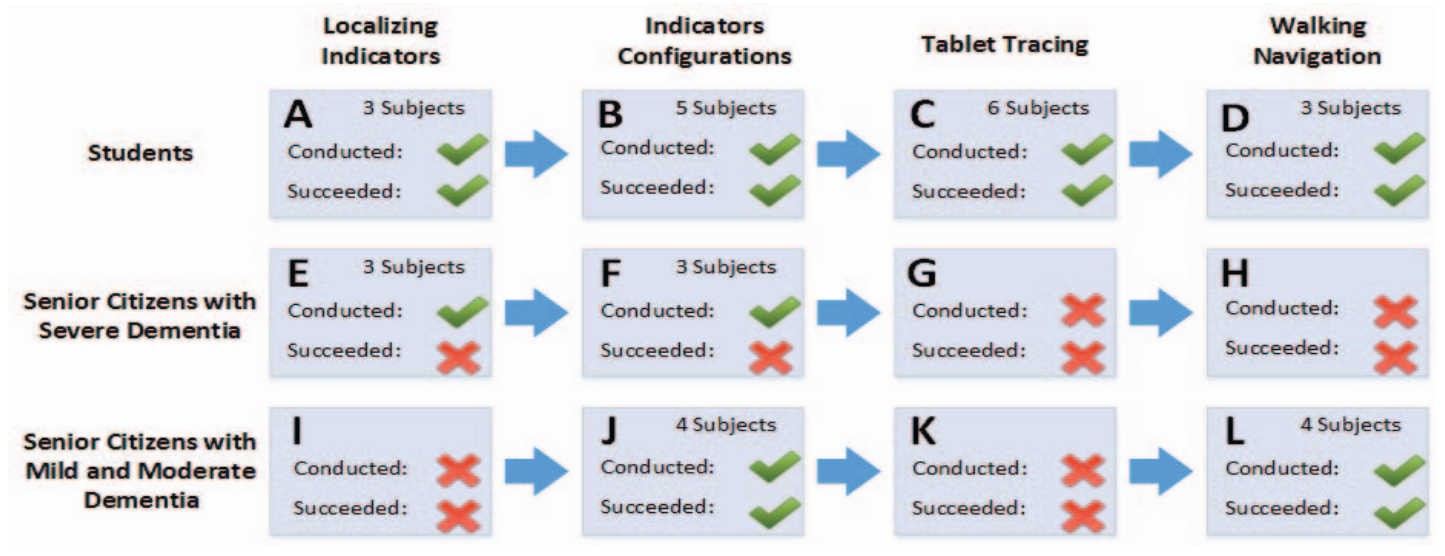

Fig. 8. It shows the sequence and the number of subjects in all the experiments. It indicates the conducted experiments with tangible collected data to analyze. It also shows the failed experiments because of the difficulty of test procedure for the elderly users.

[5] J. G. Wilpon and C. N. Jacobsen, "A study of speech recognition for children and the elderly," in Acoustics, Speech, and Signal Processing, 1996. ICASSP-96. Conference Proceedings., 1996 IEEE International Conference on, vol. 1. IEEE, 1996, pp. 349-352.

[6] C. B. Rebola and B. Jones, "Sympathetic devices: designing technologies for older adults," in Proceedings of the 31st ACM international conference on Design of communication. ACM, 2013, pp. 151-156.

[7] B. Thomas, V. Demczuk, W. Piekarski, D. Hepworth, and B. Gunther, "A wearable computer system with augmented reality to support terrestrial navigation," in Wearable Computers, 1998. Digest of Papers. Second International Symposium on. IEEE, 1998, pp. 168-171.

[8] S. Park and S. Jayaraman, "Enhancing the quality of life through wearable technology," Engineering in Medicine and Biology Magazine, IEEE, vol. 22, no. 3, pp. 41-48, 2003.

[9] D. Sedgwick, "Will google glass distract a driver?" Automotive News, vol. 88, no. 6604, p. 90, Jan 20, 2014 2014, source type: scholarlyjournals; Object type: Article; Object type: Feature; CSAUnique: dae63da6-9c5a-4d41-b5dbcsamfg101v; AccNum: 19152820; ISSN: 0005-1551. [Online]. Available: http://search.proquest.com/docview/1506346471?accountid=13031

[10] T. Danton, "Life with google glass," PC Pro, no. 240, p. 86, Oct 2014 2014, source type: scholarlyjournals; Object type: Article; Object type: Feature; CSAUnique: 1c27fb52-f43e-49b9-9155mfgefd101; AccNum: 20599681; ISSN: 1355-4603. [Online]. Available: http: //search.proquest.com/docview/1620059966?accountid=13031

[11] K. Kunze, N. Henze, and K. Kise, "Wearable computing for older adults: initial insights into head-mounted display usage," in Proceedings of the 2014 ACM International Joint Conference on Pervasive and Ubiquitous
Computing: Adjunct Publication. ACM, 2014, pp. 83-86.

[12] "Epson moverio smart glasses," http://www.epson.com/cgi-bin/Store/jsp/ Landing/moverio-bt-200-smart-glasses.do?UseCookie $=$ yes\&oid $=0$.

[13] A. Firouzian, Z. Asghar, J. Tervonen, P. Pulli, and G. Yamamoto, "Conceptual design and implementation of indicator-based smart glasses: A navigational device for remote assistance of senior citizens suffering from memory loss," in Medical Information and Communication Technology (ISMICT), 2015 9th International Symposium on. IEEE, 2015, pp. 153-156.

[14] A. Hevner and S. Chatterjee, Design research in information systems: theory and practice. Springer Science \& Business Media, 2010, vol. 22.

[15] M. Lkk, A. Firouzian, J. Tervonen, G. Yamamoto, and P. Pulli, Attention Control and Eyesight Focus for Senior Citizens, ser. Virtual, Augmented and Mixed Reality. Applications of Virtual and Augmented Reality. Springer, 2014, pp. 309-315.

[16] S. Manel, H. C. Williams, and S. J. Ormerod, "Evaluating presenceabsence models in ecology: the need to account for prevalence," Journal of Applied Ecology, vol. 38, no. 5, pp. 921-931, 2001.

[17] M. F. Folstein, S. E. Folstein, and P. R. McHugh, "mini-mental state: a practical method for grading the cognitive state of patients for the clinician," Journal of psychiatric research, vol. 12, no. 3, pp. 189-198, 1975.

[18] G. Hubbard, M. G. Downs, and S. Tester, "Including older people with dementia in research: challenges and strategies," Aging \& Mental Health, vol. 7, no. 5, pp. 351-362, 2003

[19] J. Murphy, C. M. Gray, and S. Cox, "Communication and dementia: How talking mats can help people with dementia to express themselves," 2007. 